\title{
Digital holographic microscopy applied to measurement of a flow in a T-shaped micromixer
}

\author{
T. A. Ooms $\cdot$ R. Lindken $\cdot$ J. Westerweel
}

Received: 6 October 2008/Revised: 27 April 2009/Accepted: 8 May 2009/Published online: 5 June 2009

(C) The Author(s) 2009. This article is published with open access at Springerlink.com

\begin{abstract}
In this paper, we describe measurements of a three-dimensional (3D) flow in a $\mathrm{T}$-shaped micromixer by means of digital holographic microscopy. Imaging tracer particles in a microscopic flow with conventional microscopy is accompanied by a small depth-of-field, which hinders true volumetric flow measurements. In holographic microscopy, the depth of the measurement domain does not have this limitation because any desired image plane can be reconstructed after recording. Our digital holographic microscope (DHM) consists of a conventional in-line recording system with an added magnifying optical element. The measured flow velocity and the calculated vorticity illustrate four streamwise vortices in the micromixer outflow channel. Because the investigated flow is stationary and strongly 3D, the DHM performance (i.e. accuracy and resolution) can be precisely investigated. The obtained Dynamic spatial range and Dynamic velocity range are larger than 20 and 30, respectively. High-speed multipleframe measurements illustrate the capability to simultaneously track about 80 particles in a volumetric measurement domain.
\end{abstract}

Electronic supplementary material The online version of this article (doi:10.1007/s00348-009-0683-9) contains supplementary material, which is available to authorized users.

T. A. Ooms $\cdot$ R. Lindken $\cdot$ J. Westerweel $(\bowtie)$

Laboratory for Aero and Hydrodynamics, Delft University of

Technology, Mekelweg 2, 2628 CD Delft, The Netherlands

e-mail: j.westerweel@tudelft.nl

\section{Introduction}

In microfluidics (Stone et al. 2004), fluids are transported through channels and capillaries with a diameter less than $1 \mathrm{~mm}$. Conventionally, the flow devices have simple geometries (e.g. straight channels) in which, the fluid motion is rather trivial. With improving manufacturing capabilities, more complex geometries are generated, which leads to more complex flow patterns. To understand the complex flow patterns, it is desirable to have an instrument to measure these flows. Such an instrument is preferably able to measure the flow velocity along all three spatial axes throughout a volumetric measurement domain. A number of methods have been described for three-dimensional (3D) three-component (3C) flow velocity measurements. Maas et al. (1993) and Malik et al. (1993) used photogrammetry to perform 3D-3C flow measurements, Pereira et al. (2000) described defocusing digital particle image velocimetry, Hori and Sakakibara (2004) introduced a scanning stereoscopic particle image velocimetry system and Elsinga et al. (2006) introduced tomographic particle image velocimetry. In all the described methods, imaging is performed by lenses. Because particle images should be well focused, the depth of the measurement domain should be smaller than the depth-of-field $\left(\mathrm{DOF}^{\prime}\right)^{1}$ of

\footnotetext{
${ }^{1}$ In the field of conventional (lens-) imaging, the terms 'depth-offocus' (DOF) and 'depth-of-field' $\left(\mathrm{DOF}^{\prime}\right)$ are generally used differently than in the field of holographic imaging. In the case of conventional imaging, the term 'depth-of-field' refers to the longitudinal range in the object domain within which an object produces an image that can be considered well-focused (Eqs. 1, 2). The term 'depth-of-focus' refers to the longitudinal range over which the image plane can be translated while keeping the image in focus. In the field of holographic imaging, the term 'depth-of-focus' is generally used to indicate the longitudinal size of a diffraction-limited point-source image. The term 'depth-of-field' is used to indicate the longitudinal size of the measurement domain. This choice is adopted in this paper.
} 
the imaging system. The depth-of-field is given by Born and Wolf (1959) as:

$\mathrm{DOF}^{\prime}=\frac{\lambda}{\mathrm{NA}_{\mathrm{o}}^{2}}$

where $\lambda$ is the illumination wavelength and $\mathrm{NA}_{\mathrm{o}}$ is the numerical aperture in the object domain. ${ }^{2}$ Theory of geometrical optics predicts a relation between $\mathrm{NA}_{\mathrm{o}}$ and the $f$-number $\left(f^{\#}\right)$ of the imaging optical elements as $\mathrm{NA}_{\mathrm{o}}=$ $\left(1 /\left(2 f^{\#}\right)\right)(M /(1+M))$ where $M$ is the lateral magnification. The depth-of-field can therefore also be expressed as (Adrian 1997): ${ }^{3}$

$\mathrm{DOF}^{\prime}=4\left(1+\frac{1}{M}\right)^{2}\left(f^{\#}\right)^{2} \lambda$.

When the measured flow domain is relatively large $(M \ll 1)$ and the $f$-number has an intermediate value $\left(f^{\#} \approx 10\right)$, the depth-of-field ( $\left.\mathrm{DOF}^{\prime}\right)$ has a practical value of a few centimeters. However, in microfluidics and microscopy, the measurement domain is small which implies a high image magnification $(M \gg 1)$ and the numerical aperture is typically large (i.e. a few tenths). In this case, Eq. 2 predicts a very small DOF'. For example, an imaging system with a $1,000 \times 1,000$-pixel camera with a pixel spacing of $6.45 \mu \mathrm{m}$ and a common imaging lens (i.e. a microscope objective, $M=20, \mathrm{NA}=0.40$ ) yields $\mathrm{DOF}^{\prime}=3.4 \mu \mathrm{m}$. The corresponding transverse field-ofview is $323 \mu \mathrm{m}$, which implies an essentially flat measurement domain. We therefore consider the above methods are unsuitable for volumetric (3D) microscopic flow measurements.

Interestingly, holographic particle image velocimetry (HPIV) favors the small depth-of-focus (DOF) (see footnote 1) that is inherent to microscopic imaging because it leads to an accurate measurement of tracer particle positions along the optical axis. The depth of the measurement domain is not limited by the small DOF because planesof-focus can be selected at any given depth during the hologram-reconstruction procedure. This makes HPIV an ideal method for microscopic flow measurements. An instrument that combines microscopy and digital holography is generally referred to as a Digital Holographic Microscope (DHM). Although a significant amount of work has been published on the application of a DHM in biological research (Haddad et al. 1992; Dubois et al.

\footnotetext{
2 Although Eq. 2 is not strictly valid for high-magnification microscopy (NA $\approx 1$ ), Young et al. (1993) show that it accurately predicts $\mathrm{DOF}^{\prime}$ for the experimental parameters in this work.

${ }^{3}$ When particle images are evaluated with a correlation analysis, the 'correlation depth' (Olsen and Adrian 2000) indicates the depth of the particle field that contributes to the measurement. The correlation depth then essentially replaces $\mathrm{DOF}^{\prime}$. The correlation depth is generally close to $\mathrm{DOF}^{\prime}$.
}

1999; Xu et al. 2003) and in micro-structure research (Xu et al. 2001), only a few publications have appeared on the application of a DHM to microfluidics. Satake et al. (2005) first described the application of a DHM to flow measurements in a micro channel and later presented improved results (Satake et al. 2006). In their work, transversely oriented laminar channel flows are investigated. Such a flow allows for validation of the transverse component of the measured velocity, but not the longitudinal component which is generally most critical in digital particle holography. Sheng et al. (2006) investigated the capability of the DHM by studying the effect of several different experimental settings (i.e. magnification, particle concentration) on measurement results. Their DHM was applied to measurement of a turbulent boundary layer (Sheng et al. 2008). The types of flows investigated by Sheng et al. (i.e. Brownian motion, flows around microscopic organisms and turbulent flows) are instationary and unpredictable and are therefore relatively unsuited for the validation of the performance of a DHM measurement. The flow in this work is a stationary 3D-3C microscopic flow and it can therefore be used to investigate the resolution, accuracy and reliability of DHM measurements. The described flow is generated in the outflow channel of a T-shaped micromixer (further named T-mixer) which has been extensively studied (Gobby et al. 2001; Engler et al. 2004; Wong et al. 2004; Hoffmann et al. 2006; Lindken et al. 2006). This flow has a $3 \mathrm{D}$ structure as it contains secondary vortical flow patterns (so-called Dean vortices). Since the measured flow is time-stationary, as is often the case in microfluidic flows, the obtained velocity field of multiple time-consecutive recordings can be combined to form a single high-spatialresolution velocity field. With this choice, the spatial resolution increases dramatically, sacrificing the possibility to perform time-resolved measurements.

Additionally, high-speed multiple-exposure measurements are performed on the flow and particles are recorded in the measurement domain during multiple (typically 1030) exposures. This capability allows a Lagrangian analysis of the flow and is unique to a true volumetric flow measurement instrument such as a DHM. This capability clearly distinguishes a DHM from planar measurement methods such as microscopic PIV using stereoscopic viewing.

Section 2 describes the principle of a DHM, illustrates the common configuration of a DHM and explains why digital holography and microscopy form such a strong combination. Section 3 describes how to choose the experimental parameters in order to obtain optimal DHM performance. Section 4 describes the experimental procedure and data-analysis. Section 5 shows the measurement results which are discussed in Sects. 6 and 7. 


\section{Principle}

In digital holographic microscopy, two different approaches can be distinguished: the so-called Fourier transform approach was introduced by Haddad et al. (1992) and the convolution approach was described by Sheng et al. (2006). This paper follows the second approach, which is mainly motivated by the fact that-in contrast to the first approach - the pixel size in the reconstruction planes does not depend on the longitudinal position of the reconstruction plane which is convenient-if not essential-for a correct analysis of volumetric fluid motion.

The optical setup generally consists of a collimated light beam that illuminates an object. The scattered light (object field) and the non-scattered light (reference field) interfere in an imaginary plane that is located close to-but generally outside - the imaged object. This plane is called the 'hologram plane'. The hologram plane is magnified by an optical element (i.e. a microscope objective) and imaged onto a so-called 'hologram-image plane' where the detector of a lensless digital camera is placed. The intensity in the hologram plane $\left[I_{h}(x, y)\right]$ and the hologram-image plane $\left[I_{h i}(x, y)\right]$ are related by (Goodman 1996; Sheng et al. 2006):

$I_{h}(x, y)=M^{2} \cdot I_{h i}(-M \cdot x,-M \cdot y)$

where $M$ is the magnification of the microscope objective. Diffraction by the aperture of the microscope objective is ignored. Without magnification $(M=1)$, the NA of a typical DHPIV system is restricted to a relatively low value by the Nyquist sampling criterion. To obtain proper sampling of the hologram, the optical numerical aperture (NA) should not exceed $\left(\lambda / 2 d_{r}\right)$, where $d_{r}$ is the camera pixel spacing. In practice, this limits the NA to a value of a few hundredths. This leads to a large particle image depth-offocus $\left(\mathrm{DOF}_{p i}\right)$ compared to the transverse size of a particle image and consequently to a relatively large inaccuracy of measured longitudinal velocity (Ooms et al. 2006). This problem could be overcome if cameras were available with a pixel spacing of the order of the illumination wavelength. However, such cameras are not available and an alternative solution is needed: a high-NA hologram with spatial structures as small as $\lambda_{0}$ can be magnified with an optical element onto the digital camera (Fig. 1). The size of the smallest structures of the hologram are hereby increased by a factor $M$ and proper hologram sampling can be performed with a typical digital camera.

The subsequent numerical reconstruction is based on the 'original hologram' $I_{h}(x, y)$, which is derived from the recorded 'hologram image' $I_{h i}(x, y)$ by (3). The numerical reconstruction is performed by convoluting $I_{h}(x, y)$ with the Rayleigh-Sommerfeld diffraction term (Goodman 1996)

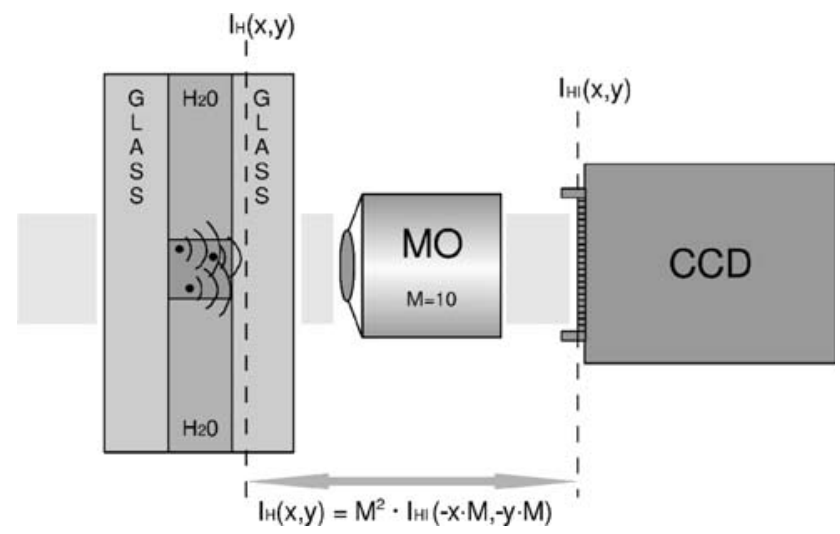

Fig. 1 Schematic configuration of a digital holographic microscope

$K_{z}(x, y)=\frac{1}{i \lambda_{0}} \frac{\exp \left(i k \sqrt{x^{2}+y^{2}+z^{2}}\right)}{\sqrt{x^{2}+y^{2}+z^{2}}} \cos \theta$.

In this expression, $z$ is a selected distance from the hologram plane, $\quad k=2 \pi / \lambda_{0}$ and $\cos \theta$ equals $z / \sqrt{x^{2}+y^{2}+z^{2}}$. This leads to a $3 \mathrm{D}$ numerical image $I_{z}(x, y)$ of the particle field whose transverse size is equal to the size of the hologram.

After hologram reconstruction, a particle tracking algorithm is applied to determine individual particle positions and particle displacements between successive recordings. With a known time-delay between two recordings, a 3D volume with up to a few hundred velocity vectors can be obtained. In case of a stationary flow, these velocity fields can be combined to create an even denser vector field.

\section{Parameters}

The experimental design of a DHM in combination with a microscopic flow requires an appropriate choice of many (interrelated) parameter values. This process is discussed in this section.

It should be mentioned that this analysis implies several simplifications, and should be considered as an initial systematic approach for the selection of the parameter values. Primarily, it may be limited to small NA (i.e. when a paraxial approximation would still be valid); Sheng et al. (2006) compare the performance of systems with low and high (effective) NA.

The transverse field-of-view (FOV) is given by:

$\mathrm{FOV}=\frac{N_{x, y} \cdot d_{r}}{M}$

where $N_{x, y}$ equals the number of horizontal or vertical pixels on the camera chip. 
A main challenge of digital holography is to obtain an acceptable accuracy of the measured longitudinal velocity. Maximization of the effective NA of the optical system is essential to meet this goal. In our setup, five factors are identified that mainly determine the effective NA. The first factor is the particle scattering profile. Particle scattering in the forward direction is generally dominated by diffraction. The particle scattering profile is modeled by the diffraction pattern of a transversely oriented circular opaque disc with a diameter that equals the particle diameter (Goodman 1996). The resulting profile in the far-field is referred to as an Airy pattern, which consists of a high-intensity central lobe surrounded by concentric fringes. The half-width of the central lobe $\left(d_{\text {Airy }}\right)$ equals $1.22 \lambda z / d_{\mathrm{p}}$ (Goodman 1996), where $\lambda$ is the illumination wavelength inside the fluid, $z$ is the longitudinal distance between the particle and the hologram and $d_{\mathrm{p}}$ is the particle diameter. These are related to a corresponding angular aperture $\left(\phi_{\text {part }}\right)$ as $d_{\text {Airy }} /$ $z=\tan \left(\phi_{\text {part }}\right)$. The approximation $\tan \left(\phi_{\text {part }}\right) \approx \sin \left(\phi_{\text {part }}\right)$ leads to an expression of the particle-contribution to the NA (defined as $n \sin (\phi)$ (Hecht 2002), where $n$ is the index of refraction):

$\mathrm{NA}_{\text {part }}=n_{w} \cdot 1.22 \frac{\left(\lambda_{0} / n_{w}\right)}{d_{\mathrm{p}}}=1.22 \frac{\lambda_{0}}{d_{\mathrm{p}}}$

where $n_{w}$ is the fluid (i.e. water) index-of-refraction and $\lambda_{0}$ is the illumination wavelength in vacuum. The second factor that influences the effective NA is the channel geometry. Propagation of high-NA light rays could be obstructed by channel walls. The effect on the NA is estimated by considering a particle in the channel, at a central transverse position and near the front channel wall, as shown in Fig. 2. Evaluating a particle at this position is expected to represent a worst-case scenario. It is clear that the angular range of the scattered light rays is limited to $\phi_{\text {geom }}$, as shown in Fig. 2.

Using the approximation $\tan \left(\phi_{\text {geom }}\right) \approx \sin \left(\phi_{\text {geom }}\right)$, the so-called 'geometrical limitation' of the NA is:

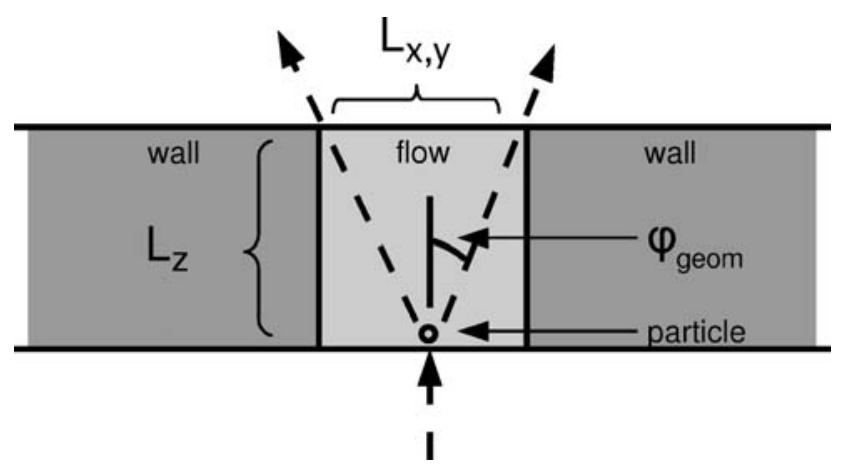

Fig. 2 Effect of the channel shape on effective NA

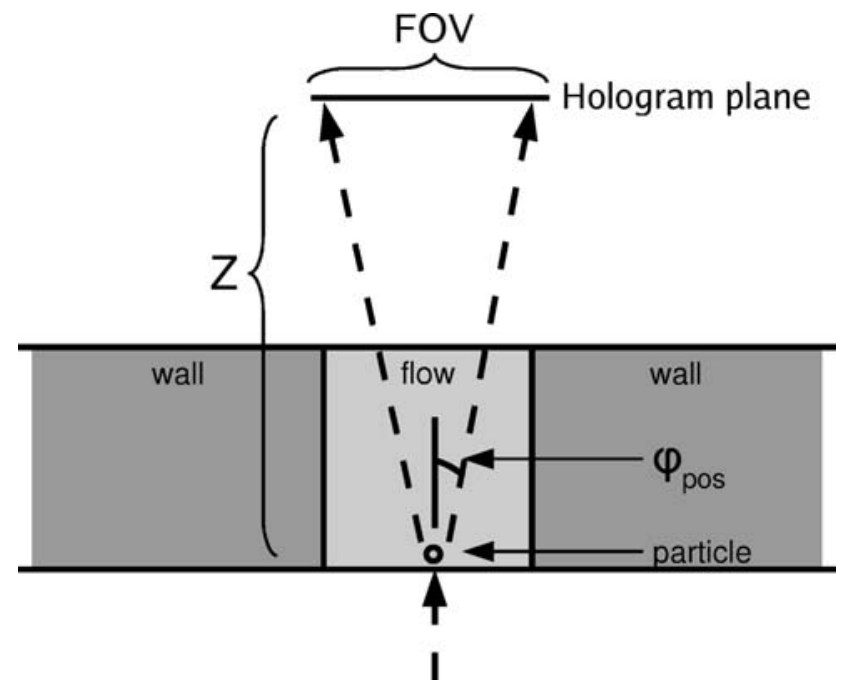

Fig. 3 Effect of hologram-plane position on effective NA

$\mathrm{NA}_{\text {geom }}=n_{w} \cdot \frac{L_{x, y} / 2}{L_{z}}$

where $L_{x, y}$ is the transverse flow dimension ${ }^{4}$ and $L_{z}$ is the longitudinal flow dimension.

The third factor that can affect the effective NA is the position of the hologram plane.

As shown in Fig. 3, when the hologram plane is located far from the object, only a small angular range of the light is recorded. Evaluating the effect on a particle that is located in the transverse center of the channel farthest away from the hologram plane should represent a worst-case scenario. Using the approximation $\tan \left(\phi_{\mathrm{pos}}\right) \approx \sin \left(\phi_{\mathrm{pos}}\right)$, the contribution to the NA can be expressed as:

$\mathrm{NA}_{\mathrm{pos}}=n \cdot \frac{\mathrm{FOV} / 2}{z_{\max }}$

where $z_{\max }$ is the distance between the hologram plane and the most distant particle. In this expression, $n$ is a function of the index-of-refraction of the fluid and the refractive index of the rear glass plate separating the channel and the microscope objective. Because the hologram plane is located almost directly behind the flow $(100 \mu \mathrm{m})$, the effect of the index of refraction of the rear glass plate on the propagation of the light waves is neglected and $n$ is chosen equal to $n_{w}$. The microscope objective also affects the NA. Its value $\left(\mathrm{NA}_{\text {micr }}\right)$ is a fixed property as determined by the manufacturer. The final factor that influences the effective NA is the camera chip. The Nyquist sampling criterion prescribes a maximum interference angle $\phi_{\text {cam }}$ between the object field and the reference field of $\arcsin \left[\lambda_{0} /\left(2 d_{r}\right)\right]$ which corresponds to:

\footnotetext{
${ }^{4}$ Note that FOV is a property of the optics and that $L_{x, y}$ represents the flow geometry.
} 
$\mathrm{NA}_{\text {cam }}=\frac{\lambda_{0}}{2 \cdot d_{r}}$

These multiple NA-terms can be interpreted as multiple low-pass spatial-frequency filtering operations of the transverse light amplitude field and the effective NA is roughly limited by the smallest NA-term. The effective NA $\left(\mathrm{NA}_{\text {eff }}\right)$ of the whole system is considered equal to the smallest of the five NA-values:

$\mathrm{NA}_{\text {eff }} \approx \min \left[\mathrm{NA}_{\text {part }}, \mathrm{NA}_{\text {geom }}, \mathrm{NA}_{\text {pos }}, \mathrm{NA}_{\text {micr }},\left(M \cdot \mathrm{NA}_{\text {cam }}\right)\right]$.

The term $\mathrm{NA}_{\text {cam }}$ is multiplied by $M$ to account for the difference between the NA on the object side and the NA on the image side of the microscope objective.

The information capacity of a (digital) hologram can be described by the space-bandwidth product (Yamaguchi and Zhang 1997). The space-component of this product is the FOV. The available bandwidth of the digitized hologram, ranges from $-M /\left(2 d_{r}\right)$ to $+M /\left(2 d_{r}\right)$ (Nyquist criterion). Because the information capacity (space-bandwidth product) of a digital hologram is the main limitation of the performance of digital holography (Yamaguchi and Zhang 1997; Meng et al. 2004; Koek et al. 2005), it is essential to design a measurement so that the space-bandwidth capacity of the camera chip is maximally used. This implies that the FOV should not significantly exceed the transverse dimension of the imaged object. Additionally, the bandwidth (or effective NA) should preferably be limited by the last term in (Eq. 10).

With a known effective NA, the diffraction limit $\delta$ of the optical system can be calculated with:

$\delta=1.22 \frac{\lambda_{0}}{\mathrm{NA}_{\mathrm{eff}}}$

If $d_{\mathrm{p}}<\delta$, particle imaging is diffraction limited (assuming the absence of optical aberrations) and the particle image diameter $d_{p i}$ approximately equals $\delta$. The longitudinal size of a diffraction-limited particle image is given by Lommel (1885) and rewritten by Born and Wolf (1959). The longitudinal intensity profile $[I(z)]$ of a diffraction limited point-source image that is created by an optical element with a circular aperture is (Born and Wolf 1959):

$I(z)=I_{0}\left(\frac{\sin \left(\frac{\pi \mathrm{NA}^{2} \mathrm{z}}{2 \lambda}\right)}{\frac{\pi \mathrm{NA}^{2} \mathrm{z}}{2 \lambda}}\right)^{2}$

where $I_{0}$ represents the particle-image intensity in the focus point. Using this function, a $20 \%$ intensity loss w.r.t. particle focus is found at $\delta z=\lambda_{0} /\left(2 \mathrm{NA}^{2}\right)$, which leads to a depth-of-focus of $\lambda_{0} / \mathrm{NA}^{2}$. In this work, a similar term, the 'particle-image depth-of-focus' $\left(\mathrm{DOF}_{p i}\right)$ is defined as the full-width-half-maximum (FWHM) of the longitudinal intensity profile of a particle image (50\% intensity loss w.r.t. particle focus). The difference between a $20 \%$-loss and a 50\%-loss can be corrected with a factor 1.77 (calculated from Eq. 12), which leads to:

$\mathrm{DOF}_{p i}=1.77 \frac{\lambda_{0}}{\mathrm{NA}_{\mathrm{eff}}^{2}}$

When particle imaging is diffraction-limited, the $\mathrm{DOF}_{p i}$ does not depend on $d_{\mathrm{p}}$.

Because the particle density is relatively low in these experiments, hologram reconstructions are analyzed with a particle tracking velocimetry (PTV) algorithm (Adamczyk and Rimai 1988). In order to relate the appropriate particle images of the first and second time-consecutive recording, the particle displacement between the first and the second recording should be significantly smaller than the typical distance between the particles. This requirement can be mathematically expressed as:

$\Delta t \ll \frac{0.55\left(C_{p}\right)^{-1 / 3}}{v_{\max }}$

where $\Delta t$ equals the time between the two recordings, $v_{\max }$ is the maximum flow velocity, $C_{\mathrm{p}}$ is the particle concentration and $0.55\left(C_{\mathrm{p}}\right)^{-1 / 3}$ is the average distance between two nearest particles in a randomly distributed particle field (Chandrasekhar 1943).

Two parameter sets are given in Table 1. Parameter set 1 represents double-frame single-exposure measurements with a PCO 2000 camera. Parameter set 2 represents multiple-frame particle-tracking measurements with a high speed camera (Photron APX-RS). Although $\mathrm{NA}_{\text {eff }}$ in Parameter Set 1 should be limited by $\mathrm{NA}_{\text {cam }}$, it is actually limited by $\mathrm{NA}_{\text {part }}$ and $\mathrm{NA}_{\text {micr }}$. It is recommended that $\mathrm{NA}_{\text {part }}$ and $\mathrm{NA}_{\text {micr }}$ are increased in future applications to increase $\mathrm{NA}_{\text {eff }}$ by a factor $1.44\left(=\frac{0.36}{0.25}\right)$ and reduce $\mathrm{DOF}_{p i}$ by a factor $2\left(=\left(\frac{0.36}{0.25}\right)^{2}\right)$. Parameter Set 2 is well-chosen in terms of the optimization of the effective NA.

The particle-image depth-of-focus was determined experimentally at $21.6 \mu \mathrm{m}$ (Ooms 2008, Sect. 5.3). This value is in fair agreement with the value of $15.0 \mu \mathrm{m}$ that follows from the present theoretical analysis, which supports that this analysis is reasonably accurate.

\section{Method}

\subsection{Experimental procedure}

\subsubsection{Optics}

The optical setup is similar to the setup described by Sheng et al. (2006). Illumination is generated by a pulsed 
Table 1 Parameter values

\begin{tabular}{|c|c|c|}
\hline Parameter & Parameter set 1 & Parameter set 2 \\
\hline Camera & PCO 2000 & Photron APX-RS \\
\hline$N_{x, y}$ & 2,048 & 1,024 \\
\hline$d_{r}$ & $7.40 \mu \mathrm{m}$ & $17.0 \mu \mathrm{m}$ \\
\hline$M$ & 10 & 20 \\
\hline FOV & $1.52 \times 1.52 \mathrm{~mm}^{2}$ & $0.87 \times 0.87 \mathrm{~mm}^{2}$ \\
\hline$\lambda_{0}$ & \multicolumn{2}{|c|}{$0.527 \mu \mathrm{m}$} \\
\hline$n_{w}$ & \multicolumn{2}{|c|}{1.33} \\
\hline$L_{x, y}$ & \multicolumn{2}{|c|}{$1.0 \mathrm{~mm}$} \\
\hline$L_{z}$ & \multicolumn{2}{|c|}{$1.0 \mathrm{~mm}$} \\
\hline$z_{\max }$ & \multicolumn{2}{|c|}{$1.1 \mathrm{~mm}$} \\
\hline$d_{\mathrm{p}}$ & \multicolumn{2}{|c|}{$2 \mu \mathrm{m}$} \\
\hline $\mathrm{NA}_{\text {part }}$ & 0.32 & 0.32 \\
\hline $\mathrm{NA}_{\text {geom }}$ & 0.67 & 0.67 \\
\hline $\mathrm{NA}_{\mathrm{pos}}$ & 0.92 & 0.53 \\
\hline $\mathrm{NA}_{\text {micr }}$ & 0.25 & 0.40 \\
\hline$M \cdot \mathrm{NA}_{\mathrm{cam}}$ & 0.36 & 0.31 \\
\hline $\mathrm{NA}_{\mathrm{eff}}$ & 0.25 & 0.31 \\
\hline$\delta$ & $2.6 \mu \mathrm{m}$ & $2.1 \mu \mathrm{m}$ \\
\hline $\mathrm{DOF}_{p i}$ & $15.0 \mu \mathrm{m}$ & $9.7 \mu \mathrm{m}$ \\
\hline
\end{tabular}

double-cavity Nd:YLF laser (New Wave, Pegasus). The illumination wavelength is $527 \mathrm{~nm}$, the polarization is oriented vertically with respect to the sensor, the maximum pulse energy is $10 \mathrm{~mJ}$ of which only a fraction (estimated at $0.10 \mu \mathrm{J}$ ) is used. As shown in Fig. 4, the beam passes a spatial filter which consists of a lens (L2), a pinhole ( $\mathrm{PH})$ and a plano-convex lens (L1).

After the beam is redirected by two mirrors, it passes through the T-mixer. The front and rear surfaces of the micro channel are high-quality glass plates to minimize deformation of the light beam. After the light passes through the object domain, the scattered light (object field) and the unscattered light (reference field) interfere in the hologram plane located 50-100 $\mu \mathrm{m}$ behind the flow (Fig. 1). The hologram is magnified by a microscope objective and imaged onto a digital camera. A PCO 2000 camera $(2,048 \times 2,048$ pixels, $7.4 \mu \mathrm{m}$ pixel spacing, 14-bit gray level quantization) was used for double-frame single-exposure recordings. An Olympus $M=10, \mathrm{NA}=0.25$ infinity corrected microscope objective was used with this camera for an optimal field-of-view. For a second set of measurements (high-speed multiple-frame particle-tracking), a Photron APX-RS high speed camera $(1,024 \times 1,024$ pixels, $17 \mu \mathrm{m}$ pixel spacing, 10-bit gray level quantization and $3,000 \mathrm{~Hz}$ maximum full-frame recording rate) camera was used. With this camera a Zeiss $M=20, \mathrm{NA}=0.40$ longdistance Achroplan microscope objective is used for a sufficiently large $\mathrm{NA}_{\mathrm{cam}}$.

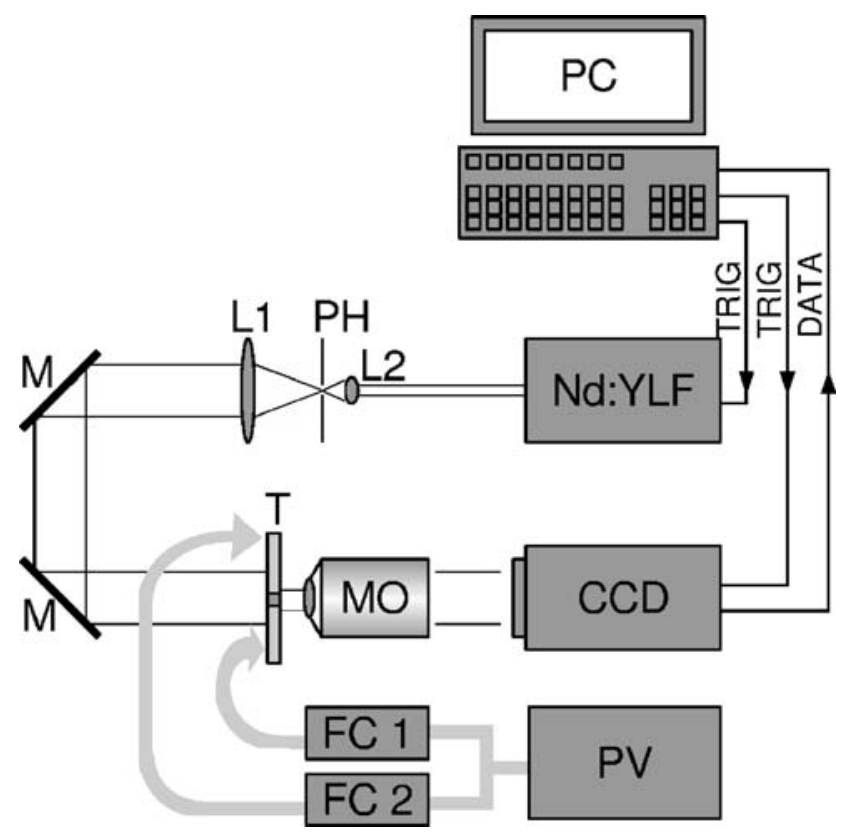

Fig. 4 Schematic of setup. $N d: Y L F$ represents the laser, $L 1$ and $L 2$ are positive lenses, $P H$ is a pinhole, $M$ are mirrors, $T$ is a T-mixer, $M O$ is a microscope objective, $C C D$ is a camera, $F C 1$ and $F C 2$ are flow controllers and $P V$ is a pressure vessel

In the double-frame measurements, 50 time-consecutive holograms were recorded at $4 \mathrm{~Hz}$. The time-delay $(\Delta t)$ between two pulses ranged between 20 and $60 \mu$ s to obtain a typical maximum in-plane particle displacement of 10 pixels. In the multiple-frame measurements, 100 timeconsecutive holograms were recorded at $3,000 \mathrm{~Hz}$. At this recording frequency, the typical maximum in-plane particle displacement was $20-50$ pixels.

\subsubsection{Flow}

Measurements are performed on a T-mixer (Figs. 5, 10), which is essentially a junction of three rectangular channels. The fluid enters through two horizontal channels and exits via a vertical channel. The three channels have a cross-section of $1 \times 1 \mathrm{~mm}^{2}$ and are constructed by mechanically pressing two high-quality glass plates (front and rear window) against a central perspex plate with two brass plates (Fig. 5). A T-shape channel is milled in the 1mm thick perspex spacer. It should be noted that this creates a significant wall roughness on the 'side walls'. The front glass window contains three drilled 1-mm-diameter holes for the fluid inflow and outflow.

A system of a stainless-steel pressure vessel and two flow controllers (Bronkhorst, LIQUI-FLOW, L2C2) provides a constant mass flow to the inflow channels (Fig. 4) (typically $200-500 \mathrm{~g} \mathrm{~h}^{-1}$ per inflow channel). The fluid is demineralized water seeded with spherical polystyrene tracer particles with a diameter of $2 \mu \mathrm{m}$ (Micromod, 


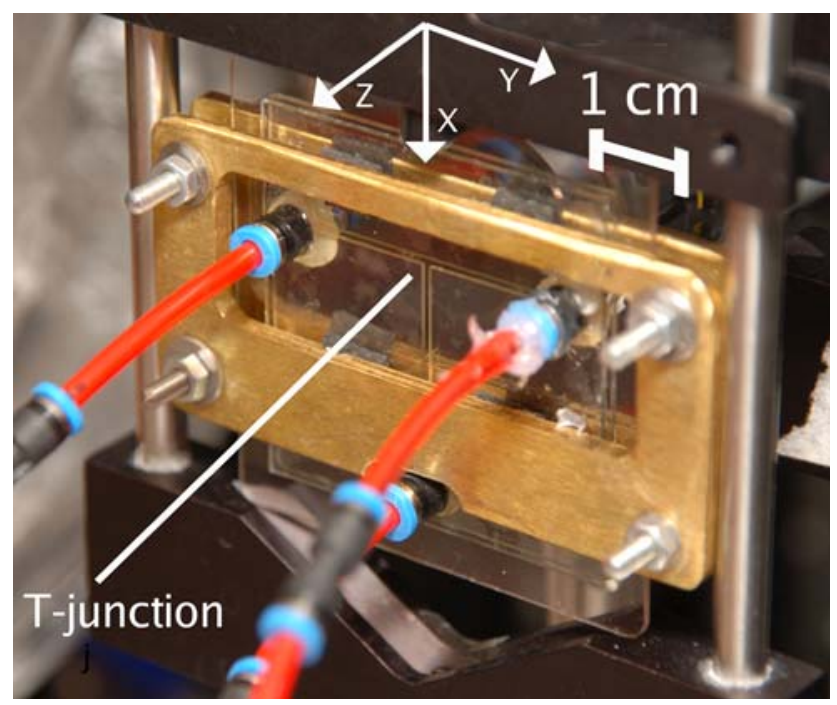

Fig. 5 Photograph of the T-mixer

Micromer, 01-54-203) and a PEG300 coating to avoid attachment to channel walls.

\subsection{Data analysis}

The data analysis procedure consists of several steps: first, noise that originates from stationary objects (i.e. dust) on optical windows is removed by subtracting an appropriate time-average hologram from all holograms. Then the hologram is reconstructed to a 3D particle-image intensityfield, which is followed by a particle-identification step which leads to a data-set of particle positions. The 'doubleframe' measurements are interrogated by a PTV analysis where displacement vectors are derived from particle image pairs. The resulting velocity vector fields of 50 recordings are combined to form a single time-averaged high-resolution vector field. These vectors are then interpolated on a regular 3D grid that forms a basis for the computation of the vorticity. These steps are described in detail in the following subsections.

\subsubsection{Time-average subtraction}

A significant amount of noise in the hologram originates from light that scatters from stationary objects (i.e. primarily from dust on optical windows) that interferes with the reference field. Because these objects are time-stationary, the unwanted signal can be removed by subtracting a time-averaged hologram from each individual hologram (Sheng et al. 2006; Ooms 2008). Figure 6 shows the same part of a typical hologram before and after subtraction of the time-averaged hologram.

Two histograms in Fig. 7 show the gray scale distribution of the two holograms in Fig. 6. The full-width-half- maximum (FWHM) of the left histogram peak is slightly more than 200, while the FWHM of the right peak is slightly more than 50 . This illustrates that $3 / 4$ of the directly recorded hologram is discarded as noise, which underlines the importance of this step to obtain an acceptable signal-to-noise ratio (SNR) in the hologram reconstruction (Fig. 7).

\subsubsection{Hologram reconstruction}

The digital hologram is reconstructed by virtually exposing it to a plane-wave reconstruction field. The hologram intensity is now regarded as a spatially varying amplitude transmittance function $t_{k}(x, y)$. The subscript $k$ is a timecounting integer. The reconstructed intensity $I_{z, k}(x, y)$ is calculated by convoluting the hologram intensity with the Rayleigh-Sommerfeld diffraction term (Eq. 4) and taking the absolute square: ${ }^{5}$

$I_{z, k}=\left(t_{k} \otimes K_{z}\right) \cdot\left(t_{k} \otimes K_{z}\right)^{*}$

where $\otimes$ represents a $2 \mathrm{D}$ convolution and $*$ represents the complex conjugate. The convolution is implemented by multiplying the 2D FT of the hologram with the 2D FT of Eq. 4 after which the inverse FT is taken of this product. Before the FT of Eq. 4 is taken, a virtual square-shaped iris is imposed on the Rayleigh-Sommerfeld term to suppress the formation of ghost images (Coupland 2004). The cutoff frequency of the iris is the Nyquist sampling frequency corresponding to the pixel resolution of the image sensor.

The size of the iris increases in proportion to the distance of the hologram to the reconstruction plane.

In this work, planes are reconstructed at 100 longitudinal positions between 0 and $1,000 \mu \mathrm{m}$ (step size $10 \mu \mathrm{m}$, uncorrected for the index-of-refraction of the fluid). A transverse slice of the reconstruction of Fig. 6 right is shown in Fig. 8.

\subsubsection{PTV}

The first step of the particle tracking algorithm is to reduce the extreme amount of data of a hologram-pair reconstruction. In this work, a single hologram reconstruction consists of $100 \times 1,024 \times 1,024$ voxels. This corresponds to 1.6 gigabytes of data per hologram-pair. In a reconstruction volume, the relevant real particle images are relatively bright, while the rest of the volume is filled with relatively low-intensity speckle. Additionally, the real

\footnotetext{
${ }^{5}$ Because of the relatively high NA in digital holographic microscopy, using the Fresnel-approximation is not permitted in this case (Goodman 1996). Reconstruction with the Rayleigh-Sommerfeld diffraction term was implemented such that its computational effort is almost equal to that of a reconstruction using the Fresnel diffraction term; see Ooms (2008).
} 

hologram before (left) and after (right) time-average subtraction. $C_{\mathrm{p}}=240 \mathrm{~mm}^{-3}$
Fig. 6 An identical part of a
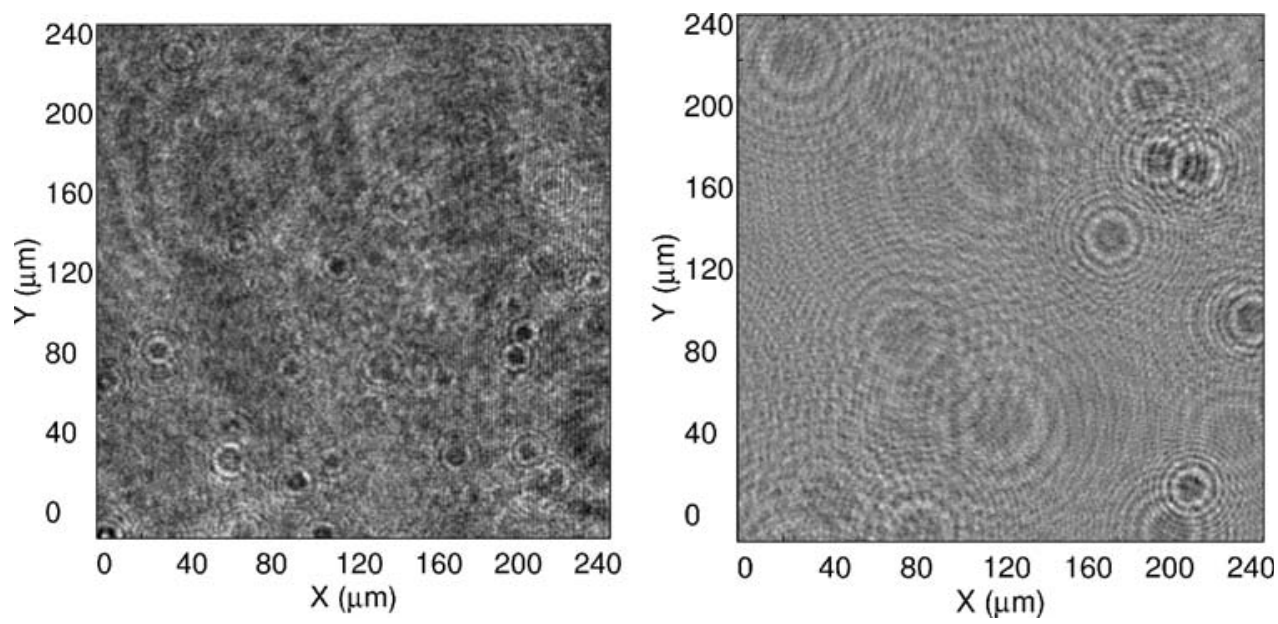

Fig. 7 Gray value distributions of Fig. 6 left and right, respectively. Please note the different scale of the two horizontal axes

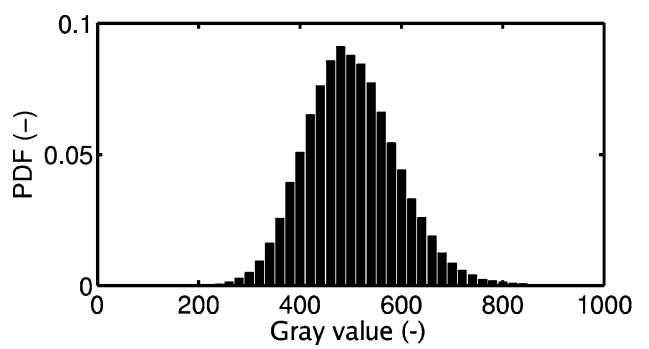

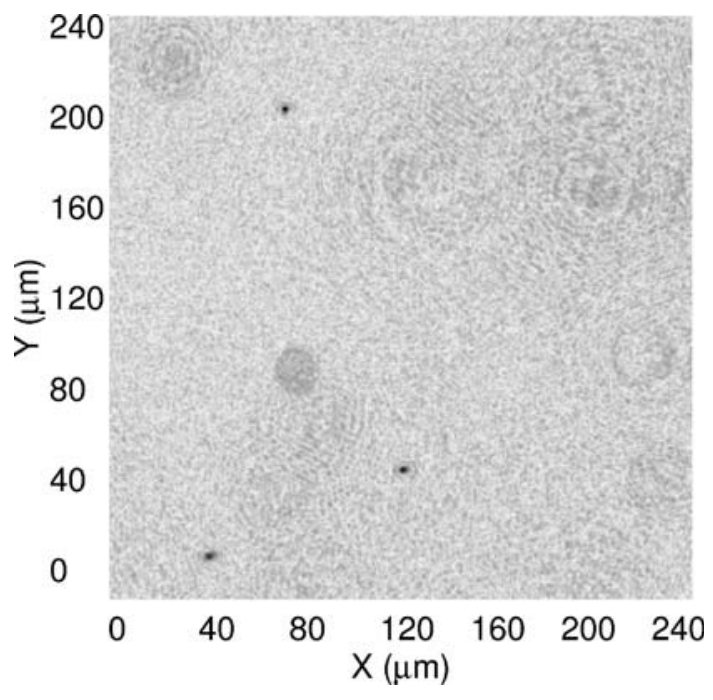

Fig. 8 A transverse slice of the reconstruction of the hologram in Fig. 6 right. The gray scale has been inverted and exaggerated to also show the relatively weak out-of-focus real particle images and the virtual particle images. The three dark points are high-intensity real particle images

particle images are sparsely distributed in space. This suggests the use of an intensity-threshold method that discards data below an intensity level $I_{\text {thres }}$ whose value is chosen taking into regard the reconstruction SNR. All points where $I(x, y, z)>I_{\text {thres }}$ are stored in a $(x[p], y[p]$, $z[p], I[p])$ array, where $p$ is a counting integer, $(x[p], y[p]$, $z[p])$ is the $3 \mathrm{D}$ integer-pixel position and $I[p]$ is the reconstructed intensity. This method reduces a typical hologram reconstruction from $10^{8}$ to $10^{3}-10^{4}$ image data points.

At this stage, a single recorded particle still corresponds to multiple data points in the $(x[p], y[p], z[p], I[p])$ data array. This is mainly caused by the relatively large longitudinal size of a reconstructed particle image. Figure 9 shows a part of the $3 \mathrm{D}$ reconstruction of the hologram in Fig. 6 right. Both the gray and black points indicate where $I(x, y, z)>I_{\text {thres. }}$.

The next step reduces the $(x[p], y[p], z[p], I[p])$ data set to 1 data point per particle. Of the various points that represent a single particle image, only the point closest to the physical particle position should remain. It is assumed that this is the point with the highest local intensity. This idea is schematically illustrated in Fig. 9, where the gray points are discarded and the black points remain.

The next step is particle pairing. Around each particle image that originates from the first exposure recording, an interrogation volume $W_{I}[=(80 \times 80 \times 80) p x=(51.6 \times$ $\left.51.6 \times 106.4) \mu^{3}\right]$ is considered. In each interrogation volume, particle images are searched that originate from the second-exposure recording. By subtracting the position of a detected second-exposure particle image from the position of a corresponding first-exposure particle image, an integer-pixel displacement vector is obtained. The obtained integer-pixel displacement vectors have a 


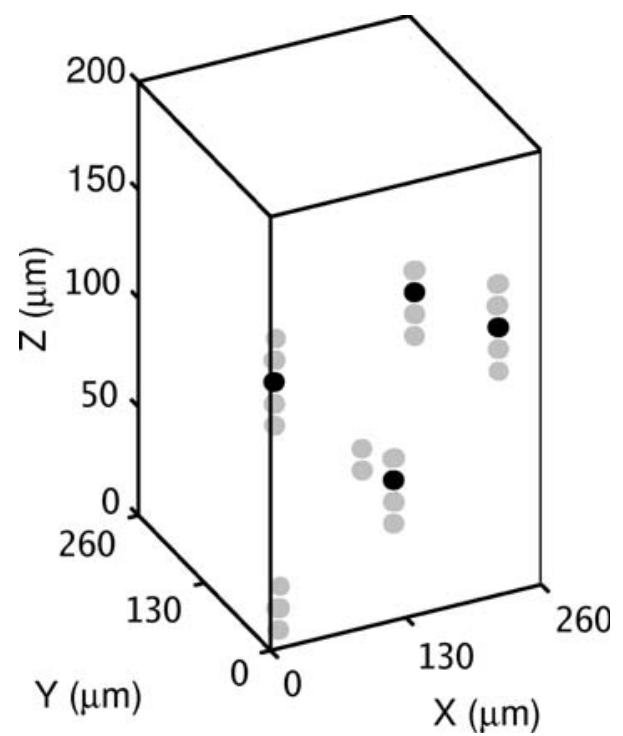

Fig. 9 After hologram reconstruction a threshold operation conserves only the data with a sufficiently high intensity. This data is indicated by gray and the black points. A second step conserves only the data points with the highest local intensity and discards all other points. The conserved (black) points are an integer-pixel estimate of the 3D particle position

transverse resolution of $d_{r} / M$ and a longitudinal resolution of $10 \mu \mathrm{m}$ (distance between reconstruction planes). A subpixel displacement is obtained by correlating local intensity data around a first-exposure particle image with local intensity data around the corresponding second-exposure particle image. A 1D Gaussian function is fitted to the correlation result to extract a sub-pixel displacement. The longitudinal position- and the longitudinal magnitude of the measured velocity vectors are corrected for the fluid index-of-refraction by multiplying these two parameters by the fluid index-of-refraction.

Then follows a vector filtering operation: a vector is rejected if the measured velocity is outsize the known limits of the flow velocity. Additionally, when multiple vectors appear to originate from the same particle image, only the smallest vector is assumed to be a correct representation of the local flow velocity and the other vectors are rejected by the filtering operation.

Each recorded hologram-pair leads to a PTV vector field with typically a few hundred vectors. These vector fields are combined to form a high spatial-resolution vector field of typically 15,000 vectors in this case.

\subsubsection{Conversion to regular grid}

To improve the accuracy of the measured velocity and to enable calculation of the vorticity, the randomly located PTV vectors are spatially binned onto a regular 3D spatial grid: a grid is defined with spacing $\left(x_{g}, y_{g}, z_{g}\right)=(100,50$, 50) $\mu \mathrm{m}$. The value of $x_{g}$ is chosen slightly larger in this case because the spatial gradient of the flow velocity is relatively small in the $x$-direction compared to the other two spatial directions. In order to calculate a vector at a grid point, a box is considered with the corresponding grid point at its center and size $\left(2 x_{g}, 2 y_{g}, 2 z_{g}\right)$. The PTV vectors that fall within the box are median-filtered. The chosen grid spacing and box size lead to a $50 \%$ overlap of adjacent boxes. The $x$-component of the vorticity of the flow $\left(\omega_{x}\right)$ is estimated by:

$$
\begin{aligned}
\omega_{x}(x, y, z)= & \frac{v_{z m}\left(x, y+y_{g}, z\right)-v_{z m}\left(x, y-y_{g}, z\right)}{2 y_{g}} \\
& -\frac{v_{y m}\left(x, y, z+z_{g}\right)-v_{y m}\left(x, y, z-z_{g}\right)}{2 z_{g}}
\end{aligned}
$$

where $v_{y m}$ and $v_{z m}$ represent the measured velocity. The $y$-component and the $z$-component of the vorticity are calculated with comparable algorithms.

The data-analysis is performed on a personal computer (CPU: AMD 2.5 GHz; OS: Linux Red Hat), using Matlab 7. Processing a set of 50 hologram pairs (time-average subtraction, reconstruction, PTV, conversion to regular grid) takes several days.

\section{Experimental results}

\subsection{Double-frame measurements}

To experimentally illustrate that a DHM is capable of true $3 \mathrm{D}$ velocity measurements, a stationary strongly $3 \mathrm{D}$ flow is investigated in a microscopic T-mixer. The flow at this Reynolds number is characterized by four Dean vortices that are created by the $90^{\circ}$ bends from the inflow channels (Wong et al. 2004). The experimental parameter values are as given by Parameter Set 1 in Table 1. Additional parameter values are: $C_{\mathrm{p}}=240$ per $\mathrm{mm}, \Delta t=40 \mu \mathrm{s}$ and the outflow-channel Reynolds number is 194 . The measurement domain is located at the beginning of the outflow channel as shown in Fig. 10.

Recording 50 time-consecutive holograms leads to a total of 18,348 vectors. This agrees well with the expected number $C_{\mathrm{p}} \times \mathrm{FOV}_{x} \times L_{y} \times L_{z} \times P=240 \mathrm{~mm}^{-3} \times$ $1.52 \mathrm{~mm}^{3} \times 50=18,240$, where $P$ is the number of evaluated hologram pairs. FOV $_{x}$ limits the size of the measurement domain in the streamwise $x$-direction and $L_{y}$ in the spanwise $y$-direction. Converting the vectors to a regular grid, as described in Sect. 4.2.4, gives visualization of the vector field at any $(x y-, y z-$ or $x z-)$ plane in the measurement volume. Four $y z$-velocity profiles are plotted in Fig. 11. This clearly shows the four streamwise vortices. 


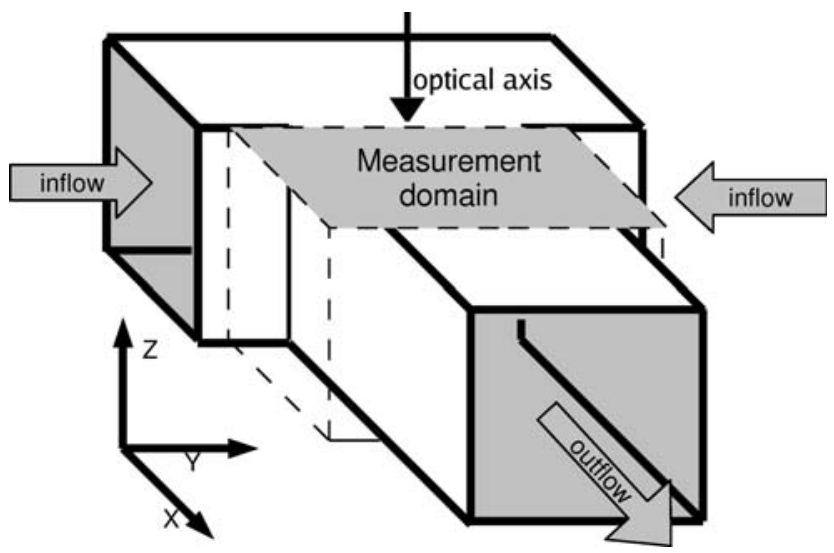

Fig. 10 The measured volume is located at the beginning of the outflow channel

Please note that the $z$-component of the plotted velocity is the velocity along the viewing direction.

Figure 12 shows the dynamic range of the measured velocity. Both the large streamwise (x-)component of the velocity, as well as the much smaller spanwise (y-and $z$-)components of the velocity are captured. The streamwise velocity component is as large as $275 \mathrm{~mm} \mathrm{~s}^{-1}$ while spanwise velocities of $10-20 \mathrm{~mm} \mathrm{~s}^{-1}$ are measured with sufficient reliability and accuracy to visually qualify them as a fair representation of the measured flow. Reliability of velocity data is quantified and discussed in detail by Ooms (2008), where it is shown that the magnitude of $99.9 \%$ of all measured velocities falls within the known boundaries of the flow velocity (for $C_{\mathrm{p}}=240 \mathrm{~mm}^{-3}, L_{z}=1 \mathrm{~mm}$ ). This value is comparable to the typical performance of planar PIV. Velocity accuracy is discussed in Sect. 6.2.

In Fig. 13, the $y z$-velocity profile at $x=800 \mu \mathrm{m}$ is shown in combination with the local $x$-component of the vorticity. Figure 14 shows the number of PTV vectors that led to a each spatially averaged vector. Relatively few vectors are found near the left and right walls (at $y \approx$ $325 \mu \mathrm{m}$ and at $y \approx 1,325 \mu \mathrm{m}$ ). This effect can be explained as follows: light that scatters from suspended particles propagates towards the hologram plane in a diverging manner. Free propagation of the scattered light is prevented near the wall which results in a reduced intensity
Fig. 11 Measured velocity in the T-mixer outflow channel showing four vortices. Plots at a low $x$-value are located closest to the junction. The channel walls are located at $y \approx 325 \mu \mathrm{m}, y \approx 1,325 \mu \mathrm{m}$, $z \approx 150 \mu \mathrm{m}$ and $z \approx 1,150 \mu \mathrm{m}$ The plane $z=0$ is the hologram plane. The $100 \mathrm{~mm} \mathrm{~s}^{-1}$-arrow indicates a reference for the magnitude of the velocity vectors
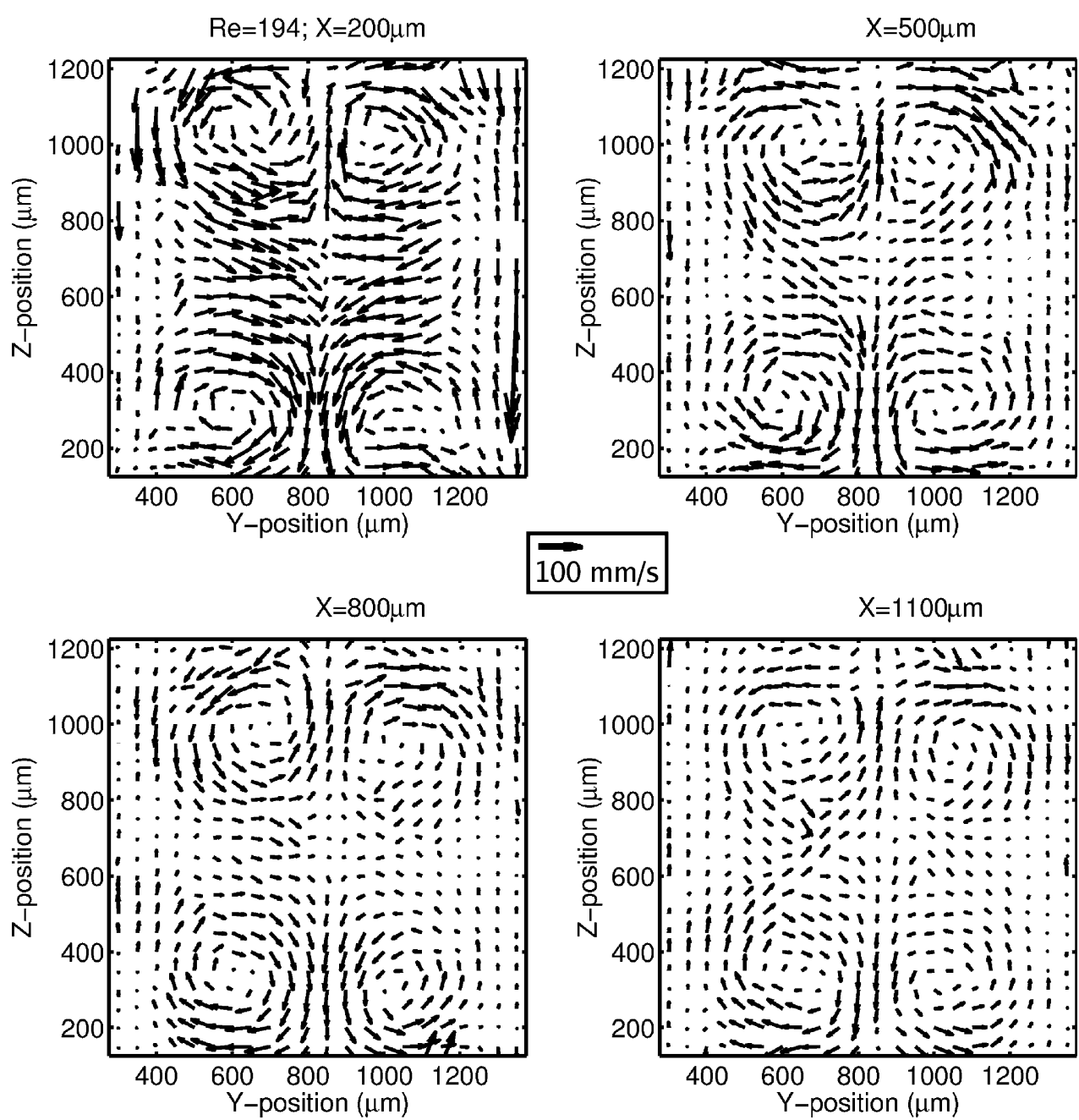


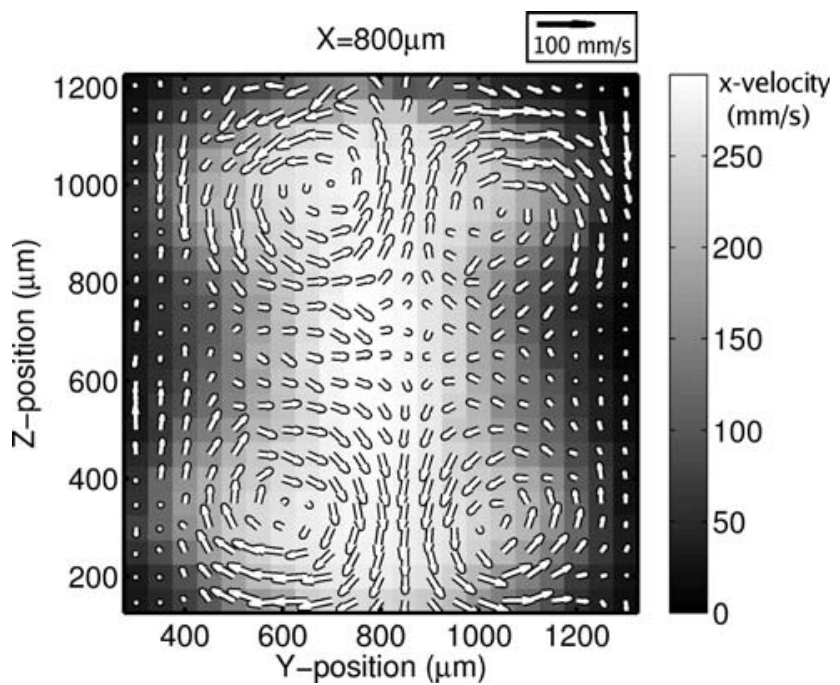

Fig. 12 Measured velocity in the T-mixer outflow channel. Vectors indicate spanwise (yz-)velocity. Gray scale indicates streamwise $(x-)$ velocity. This figure illustrates the dynamic range of the measured velocity: both the large streamwise $(x$-)velocity, as well as the much smaller the spanwise (yz-)velocities are captured. The channel walls are located at $y \approx 325 \mu \mathrm{m}, y \approx 1,325 \mu \mathrm{m}, z \approx 150 \mu \mathrm{m}$ and $z \approx$ $1,150 \mu \mathrm{m}$. The plane $z=0$ is the hologram plane. The $100 \mathrm{~mm} \mathrm{~s}^{-1}$. arrow indicates a reference for the magnitude of the velocity vectors

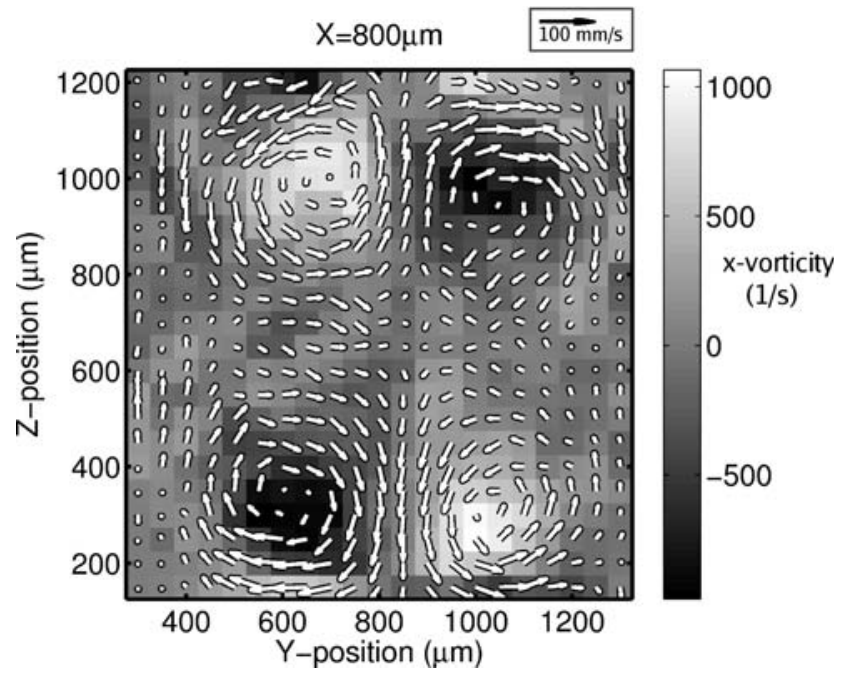

Fig. 13 Measured velocity in the T-mixer outflow channel. Vectors indicate $y z$-velocity. Gray scale indicates $x$-component of the vorticity. The channel walls are located at $y \approx 325 \mu \mathrm{m}, y \approx$ $1,325 \mu \mathrm{m}, z \approx 150 \mu \mathrm{m}$ and $z \approx 1,150 \mu \mathrm{m}$. The plane $z=0$ is the hologram plane. The $100 \mathrm{~mm} \mathrm{~s}^{-1}$-arrow indicates a reference for the magnitude of the velocity vectors

and optical aberrations in the corresponding reconstructed particle images which, in turn, reduces the chance that the particle image is detected. Near the top- and bottom wall, a reduced number of vectors per unit volume is also observed. This effect only occurs in the single top- and bottom grid layer and we expect that it is caused by the

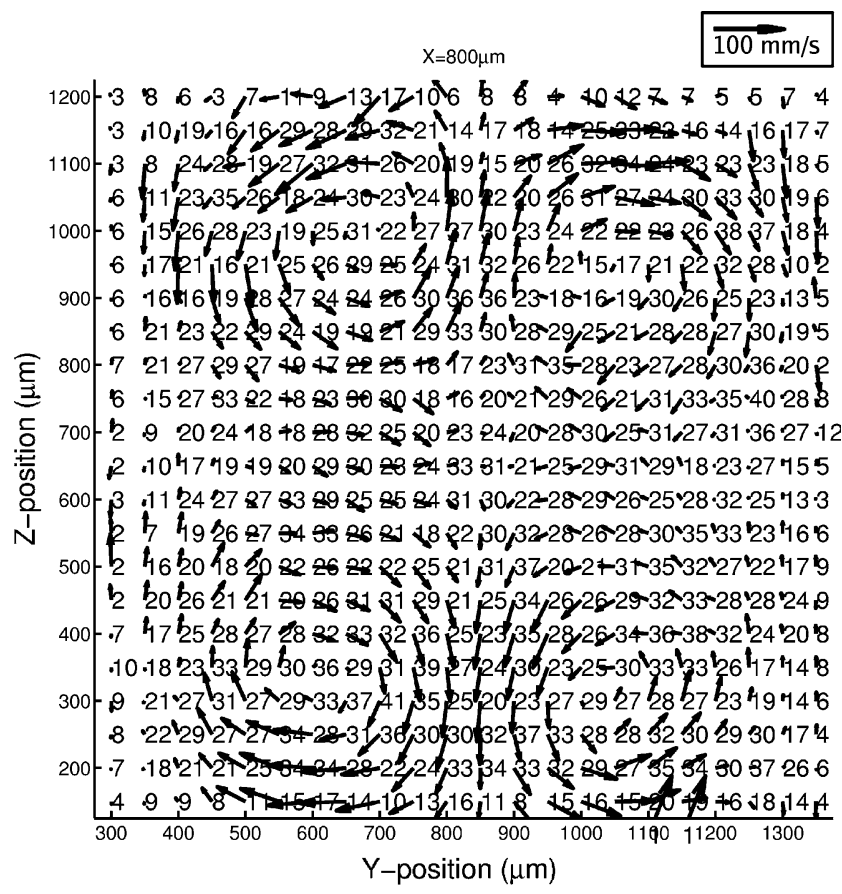

Fig. 14 Measured velocity in the T-mixer outflow channel at $x=800 \mu \mathrm{m}$. The numbers indicate the amount of PTV vectors that led to a corresponding regular-grid vector. The $100 \mathrm{~mm} \mathrm{~s}^{-1}$-arrow indicates a reference for the magnitude of the velocity vectors

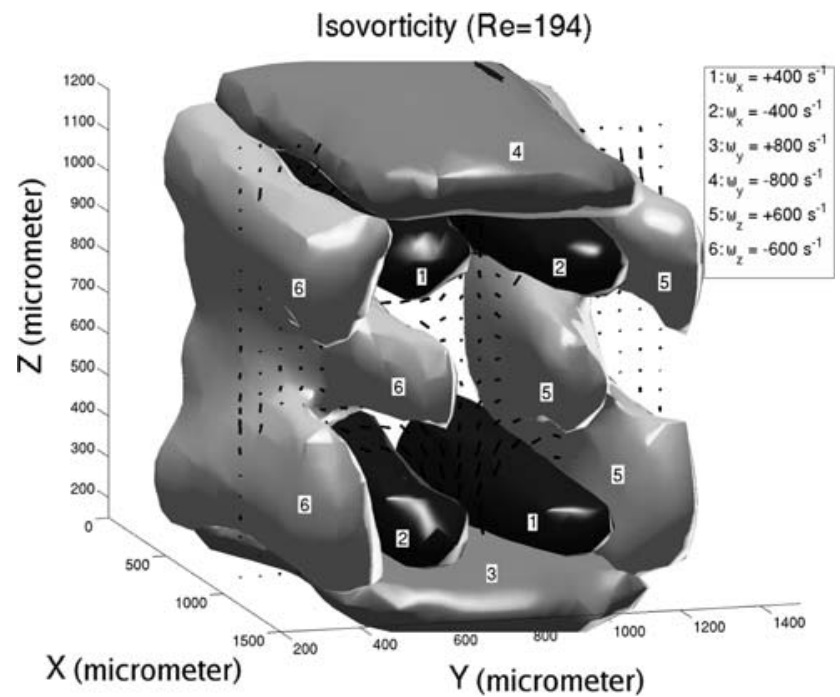

Fig. 15 Iso- $x$-vorticity, iso- $y$-vorticity, iso- $z$-vorticity surfaces and $y z$-velocity vectors at the $x=800 \mu \mathrm{m}$-plane in the T-mixer outflow channel

smaller size of these grid cells due to the presence of the top- and bottom walls. As expected, regular-grid vectors that are derived from relatively few PTV vectors (typically located near channel walls) appear less accurate.

Figure 15 shows iso- $x$-vorticity, iso- $y$-vorticity and iso$z$-vorticity surfaces. The iso- $x$-vorticity surfaces show the vorticity that is also shown in Fig. 13. A large $y$-component 
of the vorticity is found near the top- and bottom walls and probably originates mainly from the wall shear $\left(\mathrm{d} v_{x} / \mathrm{d} z\right)$. A large $z$-component of the vorticity is found near the side walls and also seems to originate from the wall shear $\left(\mathrm{d} v_{x} / \mathrm{d} y\right)$. The iso- $z$-vorticity surfaces bend towards the channel center at intermediate $z$-values.

\subsection{Multiple-frame measurements}

The capability of a DHM to follow particles in a 3D measurement domain during multiple exposures is illustrated by high-speed measurements. The experimental configuration is described in Sect. 4.1 and by Parameter Set 2 in Table 1. Other parameter values are: $C_{\mathrm{p}} \approx$ $80 \mathrm{~mm}^{-3}, \Delta t=1 / 3,000 \mathrm{~s}, R e=111$. The camera recording rate was set to the highest value and we therefore chose a lower Reynolds number to avoid extremely large frameto-frame particle-image displacements. We chose a lower $C_{\mathrm{p}}$ to ensure correct particle matching with the large frameto-frame particle-image displacement of up to 90 pixels in the $x$-direction. In the analysis of these measurements, subpixel particle-positions are calculated. The result is best visualized in an animation that is available as electronic supplementary material. Figure 16 shows a snapshot of one

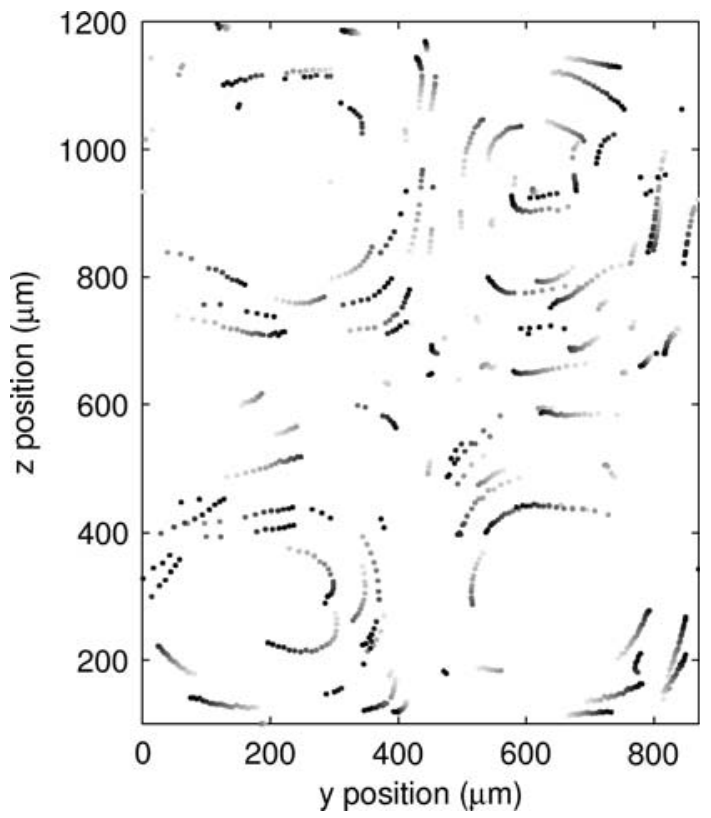

Fig. $16 y z$-projection of measured particle positions in the T-mixer outflow channel. Particle-positions of 20 time-consecutive recordings are shown to indicate particle-motion. The increasing darkness of the points indicates the recording time. The recording rate was $3,000 \mathrm{~Hz}$. The left- and right-channel walls are located just outside the shown $y$-domain. The upper and lower channel walls are located at $z \approx$ $150 \mu \mathrm{m}$ and $z \approx 1,150 \mu \mathrm{m}$. The plane $z=0$ is the hologram plane of these animations by plotting particle positions at 20 time-consecutive recordings.

\section{Discussion}

Following the approach of Adrian (1997), this section quantifies the quality of the described measurements by calculating the Dynamic spatial range (DSR) and the Dynamic velocity range (DVR). Further, the optimization of the value of $C_{\mathrm{p}}$ is discussed.

\subsection{Dynamic spatial range}

The DSR is defined as the size of the measurement domain divided by the smallest resolvable flow variation-or spatial resolution. The transverse and the longitudinal DSR are distinguished: the transverse DSR depends on the transverse size of the measurement domain which is generally determined by the optical elements (FOV). The longitudinal DSR depends on the longitudinal size of the measurement domain, which is generally determined by the depth of the flow domain $\left(L_{z}\right)$. Adrian (1997) states that the smallest resolvable spatial flow variation, or highest spatial resolution, is achieved by measurement of single particle displacements. The highest spatial resolution can therefore be estimated as the average distance between particles which equals $0.55 C_{\mathrm{p}}^{-1 / 3}$ (Eq. 14). Hence it follows that:

$\mathrm{DSR}_{x, y}=\frac{\mathrm{FOV}_{x, y} \cdot C_{\mathrm{p}}^{1 / 3}}{0.55}$

$\operatorname{DSR}_{z}=\frac{L_{z} \cdot C_{\mathrm{p}}^{1 / 3}}{0.55}$.

The DSR is maximized by maximizing the FOV, $L_{z}$ and $C_{\mathrm{p}}$. In practice, the FOV and $L_{z}$ are often determined by the investigated flow and maximization of the DSR depends mainly on the maximization of $C_{\mathrm{p}}$. In turn, $C_{\mathrm{p}}$ is limited to a maximum because increasing $C_{\mathrm{p}}$ leads to an increasing speckle noise intensity, which impairs particle-image detection (Meng et al. 1993). According to Meng et al. (2004), the ratio of the particle-image intensity $\left(I_{0}\right)$ to the mean speckle noise intensity $\left\langle I_{N}\right\rangle$ should be at least 50 to properly distinguish particle images from the speckle background. To find the value of $C_{\mathrm{p}}$ that corresponds to $I_{0} /\left\langle I_{N}\right\rangle=50$, measurements with four different values of $C_{\mathrm{p}}$ are performed and the corresponding values of $I_{0} /\left\langle I_{N}\right\rangle$ are calculated. The value of $I_{0} /\left\langle I_{N}\right\rangle$ is obtained by averaging over 100 particle images. The result is plotted in Fig. 17.

Using the criterion $\min \left(I_{0} /\left\langle I_{N}\right\rangle\right)=50$, one finds a maximum value for $C_{\mathrm{p}}$ of about $2,000 \mathrm{~mm}^{-3}$ which leadswith $\mathrm{FOV}=1.52 \mathrm{~mm}$ and $L_{z}=1 \mathrm{~mm}$ and Eqs. 17 and 18-to a maximum DSR in these experiments of: 


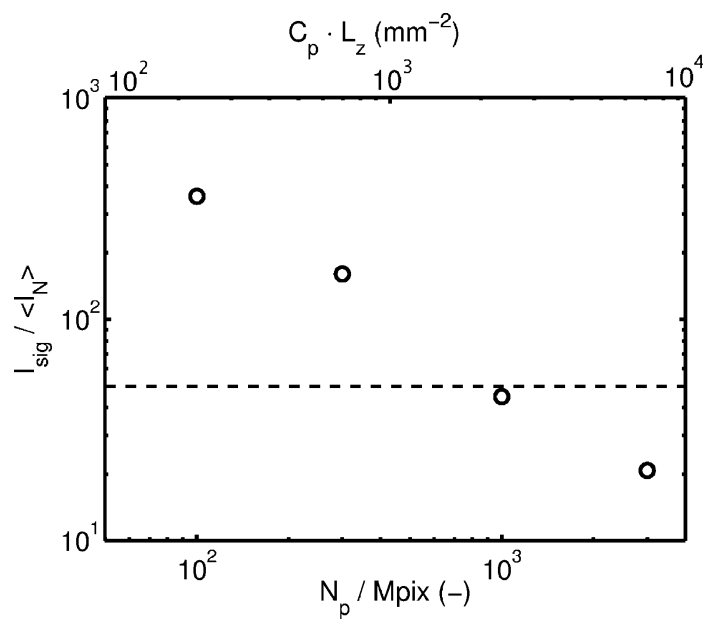

Fig. 17 The measured signal-to-noise ratio of the reconstructed image versus the particle concentration. The horizontal dashed line indicates $I_{0} /\left\langle I_{N}\right\rangle=50$ (Meng et al. 2004)

$$
\begin{aligned}
\max \left(\operatorname{DSR}_{x}\right) & =\frac{1.52 \mathrm{~mm} \cdot\left(2,000 \mathrm{~mm}^{-3}\right)^{1 / 3}}{0.55}=34.8 \\
\max \left(\operatorname{DSR}_{y, z}\right) & =\frac{1.00 \mathrm{~mm} \cdot\left(2,000 \mathrm{~mm}^{-3}\right)^{1 / 3}}{0.55}=22.9
\end{aligned}
$$

The measurement results in Sect. 5 were obtained with $C_{\mathrm{p}}=240 \mathrm{~mm}^{-3}$, which implies an obtained DSR:

$$
\begin{aligned}
\operatorname{DSR}_{x} & =\frac{1.52 \mathrm{~mm} \cdot\left(240 \mathrm{~mm}^{-3}\right)^{1 / 3}}{0.55}=17.2 \\
\operatorname{DSR}_{y, z} & =\frac{1.00 \mathrm{~mm} \cdot\left(240 \mathrm{~mm}^{-3}\right)^{1 / 3}}{0.55}=11.3 .
\end{aligned}
$$

The values of the obtained DSR are about two times lower than the found maximum obtainable DSR. The reason for the lower chosen $C_{\mathrm{p}}$ is that a high $C_{\mathrm{p}}$ negatively affects the DVR as will be shown in the next section.

As described in Sect. 9, the measured velocity vectors of multiple time-consecutive hologram pairs are combined, spatially averaged and arranged on a regular grid. After these steps, the obtained DSR is:

$$
\begin{aligned}
& \operatorname{DSR}_{x}=22 \\
& \operatorname{DSR}_{y, z}=22
\end{aligned}
$$

which is derived by counting the number of vectors along the $y$-axis and the $z$-axis in Fig. 13.

\subsection{Dynamic velocity range}

The DVR is defined by Adrian (1997) as the ratio of the maximum flow velocity to the minimum resolvable flow velocity (MRFV). The transverse MRFV can be expressed as $\gamma \mathrm{d}_{p i} / \Delta t$ (Prasad et al. 1992; Westerweel 2000), where the parameter $\gamma$ indicates the accuracy with which a particleimage's position can be determined compared to the

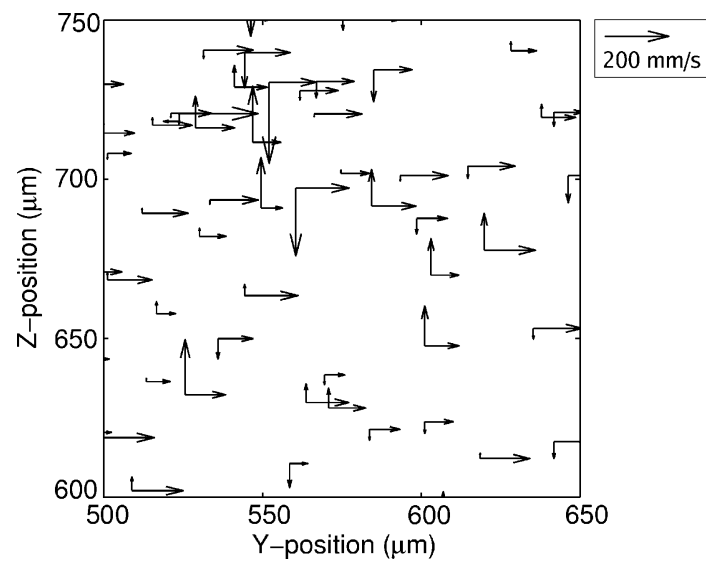

Fig. 18 A $y z$-projection of a selection of a measured velocity field with the vector $y$-component and the $z$-component separately plotted. The transverse $(y$-)component of the velocity vectors shows the rightward flow quite reliably. The longitudinal (z-)component of the velocity vectors however, has a significant random component which illustrates the inaccuracy of the measured longitudinal velocity. In this example, $C_{\mathrm{p}}$ is ten times higher than in the experiments of Sect. 5 $\left(2,400 \mathrm{~mm}^{-3}\right.$ versus $\left.240 \mathrm{~mm}^{-3}\right)$ for a clear illustration of the large inaccuracy of $v_{z m}$ compared to $v_{y m}$. The $200 \mathrm{~mm} \mathrm{~s}^{-1}$-arrow indicates a reference for the magnitude of the velocity vectors

particle image diameter. It ranges typically between 0.05 and 0.1. Similarly, the longitudinal MRFV is estimated by $\gamma \mathrm{DOF}_{p i} / \Delta t$. Hence, the expressions of the DVR are:

$\mathrm{DVR}_{x, y}=\frac{v_{\max } \cdot \Delta t}{\gamma \cdot d_{p i}}$

$\mathrm{DVR}_{z}=\frac{v_{\text {max }} \cdot \Delta t}{\gamma \cdot \mathrm{DOF}_{p i}}$

Because generally $\mathrm{DOF}_{p i}>d_{p i}$, the longitudinal DVR is the limiting factor in terms of the measured velocity accuracy and further investigation focuses only on the longitudinal DVR. This is denoted as $\mathrm{DVR}_{z}$ and is expected to depend on $C_{\mathrm{p}}$ as $\gamma$ is expected to depend on $C_{\mathrm{p}}$. The presence of the out-of-focus real images of other particles and the speckle noise that originates from the virtual images of particles reduces the accuracy of the estimated particle image position (Ooms et al. 2008). As the particle concentration increases, this effect is expected to increase. Figure 18 shows that the longitudinal DVR is especially sensitive to this effect.

The following paragraph aims to relate the value of $\mathrm{MRFV}_{z}$ to $C_{\mathrm{p}}$. The $\mathrm{MRFV}_{z}$ is determined as follows: the flow in the T-mixer $\left(R e=111, v_{\max } \approx 222 \mathrm{~mm} \mathrm{~s}^{-1}\right)$ is measured with four different values of $C_{\mathrm{p}}$ as shown in Table 2. The measurement domain is split into subvolumes with size $V_{s v}=200 \times 100 \times 100 \mu \mathrm{m}^{3}$. Of all vectors that are located inside an investigated subvolume, the standard deviation of the measured longitudinal velocity component $\sigma_{v z m}$ is calculated which is composed of the standard 
Table 2 The particle concentration, the average standard deviation of the measured $v_{z}$ and $v_{y}$ within defined subvolumes, the calculated $\mathrm{MRFV}_{z}$ (Eq. 22) and the calculated $\mathrm{DVR}_{z}$ (Eq. 20)

\begin{tabular}{lcccc}
\hline$C_{\mathrm{p}}\left(\mathrm{mm}^{-3}\right)$ & $\sigma_{\mathrm{vzm}}\left(\mathrm{mm} \mathrm{s}^{-1}\right)$ & $\sigma_{\mathrm{vym}}$ & $\mathrm{MRFV}_{z}$ & $\mathrm{DVR}_{z}$ \\
\hline 240 & 40.8 & 20 & 35.5 & 6.3 \\
720 & 48.7 & 20 & 44.4 & 5.0 \\
2,400 & 69.9 & 20 & 67.0 & 3.3 \\
7,200 & 150.1 & 20 & 148.8 & 1.5 \\
\hline
\end{tabular}

deviation of the true flow velocity $v_{z}$ inside the subvolume $\left(\sigma_{v z f}\right)$ and the standard deviation of the measurement error of $v_{z}\left(\sigma_{v z e}\right)$. We assume that both components are normally distributed around their average and statistically independent, which leads to the relation (Chatfield 1983):

$\sigma_{v z m}^{2}=\sigma_{v z f}^{2}+\sigma_{v z e}^{2}$

The term $\sigma_{v z f}$ can be calculated by focusing on the standard deviation of the measured transverse (y) velocity vectors inside a subvolume: because the inaccuracy of the measured transverse velocity is much smaller than the inaccuracy of the measured longitudinal velocity, we assume that $\sigma_{v y m}^{2} \approx \sigma_{v y f}^{2}$. Furthermore, for flow-symmetry reasons on average $\sigma_{v z f} \approx \sigma_{v y f}$ and hence the $\mathrm{MRFV}_{z}$ is estimated from Eq. 21 as:

$\mathrm{MRFV}_{z} \equiv \sigma_{v z e}=\sqrt{\sigma_{v z m}^{2}-\sigma_{v y m}^{2}}$

The plotted values of $\sigma_{v z m}$ and $\sigma_{v y m}$ in Fig. 19 and Table 2 follow from averaging over several subvolumes at different positions in the reconstructed volume. Calculated values of $\mathrm{MRFV}_{z}$ are given in Table 2. Figure 19 shows that the error of the measured $v_{z}$ increases with an increasing $C_{\mathrm{p}}$.

As described in Sect. 9, the measured velocity vectors of multiple time-consecutive hologram pairs are combined,

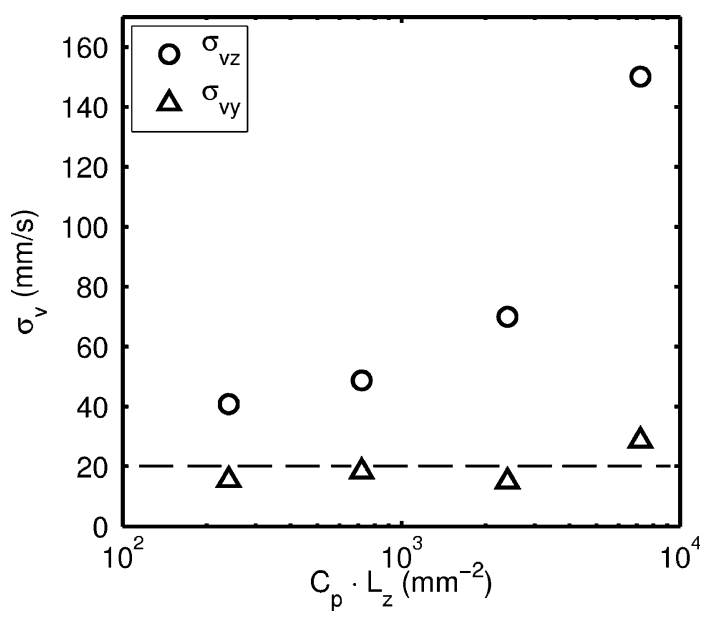

Fig. 19 The standard deviation of the measured longitudinal $(z)$ velocity and the measured transverse $(y)$ velocity versus the particle concentration spatially averaged and arranged on a regular grid. For statistically independent data, the spatial averaging is expected to reduce the inaccuracy of $v_{z}$ by $\left(N^{-1 / 2}\right)$ (Chatfield 1983), where $N$ indicates the number of vectors inside a subvolume $V_{s v}$. The value of $N$ is estimated as $N=C_{\mathrm{p}} V_{s v} P$, where $P$ indicates the number of evaluated hologram pairs. With $C_{\mathrm{p}}=240 \mathrm{~mm}^{-3}, V_{s v}=200 \times 100 \times 100 \mu \mathrm{m}^{3}$ and $P=50$ follows that $N=24$, which agrees with the observed values in Fig. 14. This suggests that the $\mathrm{DVR}_{z}$ of the T-mixer measurement results, after the vectors are arranged on the regular grid $\left(C_{\mathrm{p}}=240 \mathrm{~mm}^{-3}\right)$ is 6.3 . $\sqrt{24}=30.8$. This value agrees with Fig. 12 and is of the same order as a typical $D V R$ of conventional planar PIV.

\section{Conclusions}

A DHM is essentially an in-line digital holographic recording setup with an added magnifying optical element. An essential step of the numerical processing of the digital holograms is time-average intensity subtraction. In this work, this step discards about $3 / 4$ of the dynamic range of the original hologram as unwanted noise. After hologram reconstruction and a PTV analysis, several time-consecutive velocity vector fields are obtained. Because the recorded flow is time-stationary, time-consecutive vector fields can be combined to obtain a single vector field with improved spatial resolution containing more than 18,000 vectors for this measurement. After spatial averaging, a regular-grid vector field is obtained that clearly reveals four streamwise vortices. The corresponding transverse and longitudinal DSR equal 22, which is adequate to illustrate the vortical structures. The corresponding longitudinal DSR equals 30.8 which is within the same order of magnitude as typical 2D PIV measurements. The transverse DSR has not been investigated because it is expected to be even larger. The chosen particle concentration of $240 \mathrm{~mm}^{-3}$ is shown to be a fair compromise in terms of the obtained DSR and DVR. It is concluded that a DHM is very suited to perform three-dimensional velocity measurements of timeindependent microscopic flows with adequate spatial resolution and accuracy.

The high-speed multiple-frame measurements show that a DHM can successfully follow particles in a 3D measurement domain by recording them in multiple timeconsecutive holograms, which indicates that a DHM is suitable to study, for example, Lagrangian flow statistics of time-dependent flows. In these time-dependent measurements, the spatial-resolution is partially sacrificed $\left(\mathrm{DSR}_{z}=\right.$ $\left.\frac{1.0 \mathrm{~mm} \cdot\left(80 \mathrm{~mm}^{-3}\right)^{1 / 3}}{0.55}=7.8\right)$ to obtain an improved inaccuracy of the measured velocity. 
Acknowledgments We thank the FOM foundation for the financial support of this project, Cor Rops for providing the flow controllers and Jeanette Hussong for the useful discussions.

Open Access This article is distributed under the terms of the Creative Commons Attribution Noncommercial License which permits any noncommercial use, distribution, and reproduction in any medium, provided the original author(s) and source are credited.

\section{References}

Adrian R (1997) Dynamic ranges of velocity and spatial resolution of particle image velocimetry. Meas Sci Technol 8(12):1393-1398

Adamczyk A, Rimai L (1988) 2-Dimensional particle tracking velocimetry (PTV): technique and image processing algorithms. Exp Fluids 6(6):373-380

Born M, Wolf E (1959) Principles of optics. Pergamon Press, Oxford

Chandrasekhar S (1943) Stochastic problems in physics and astronomy. Rev Mod Phys 15(1):1-89

Chatfield C (1983) Statistics for technology: a course in applied statistics. Chapman \& Hall/CRC

Coupland J (2004) Holographic particle image velocimetry: signal recovery from under-sampled CCD data. Meas Sci Technol 15(4):711-717

Dubois F, Joannes L, Legros J (1999) Improved three-dimensional imaging with a digital holography microscope with a source of partial spatial coherence. Appl Optics 38:7085-7094

Elsinga G, Scarano F, Wieneke B, van Oudheusden B (2006) Tomographic particle image velocimetry. Exp Fluids 41:933947

Engler M, Kockmann N, Kiefer T, Woias P (2004) Numerical and experimental investigations on liquid mixing in static micromixers. Chem Eng J 101(1-3):315-322

Gobby D, Angeli P, Gavriilidis A (2001) Mixing characteristics of T-type microfluidic mixers. J Micromech Microeng 11(2):126-132

Goodman J (1996) Introduction to Fourier optics. McGraw-Hill, Singapore

Haddad W, Cullen D, Solem J, Longworth J, McPherson A, Boyer K, Rhodes C (1992) Fourier-transform holographic microscope. Appl Optics 31:4973-4978

Hecht E (2002) Optics. Addison Wesley, San Francisco

Hoffmann M, Schlüter M, Räbiger N (2006) Experimental investigation of liquid-liquid mixing in $\mathrm{T}$-shaped micro-mixers using $\mu$-LIF and $\mu$-PIV. Chem Eng Sci 61(9):2968-2976

Hori T, Sakakibara J (2004) High-speed scanning stereoscopic PIV for 3D vorticity measurement in liquids. Meas Sci Technol 15(6): 1067-1078

Koek W, Bhattacharya N, Braat J, Ooms T, Westerweel J (2005) Influence of virtual images on the signal-to-noise ratio in digital in-line particle holography. Opt Express 13(7):2578-2589

Lindken R, Westerweel J, Wieneke B (2006) Stereoscopic micro particle image velocimetry. Exp Fluids 41(2):161-171

Lommel E (1885) Die Beugungserscheinungen einer kreisrunden Oeffnung und eines kreisrunden Schirmchens. Abh Bayer Akad Math Naturwiss 15:229-328

Maas H, Gruen A, Papantoniou D (1993) Particle tracking velocimetry in three-dimensional flows. Exp Fluids 15(2):133-146
Malik N, Dracos T, Papantoniou D (1993) Particle tracking velocimetry in three-dimensional flows. Exp Fluids 15(4):279-294

Meng H, Anderson W, Hussain F, Liu D (1993) Intrinsic speckle noise in in-line particle holography. J Opt Soc Am A 10:20462058

Meng H, Pan G, Pu Y, Woodward S (2004) Holographic particle image velocimetry: from film to digital recording. Meas Sci Technol 15(4):673-685

Olsen M, Adrian R (2000) Out-of-focus effects on particle image visibility and correlation in microscopic particle image velocimetry. Exp Fluids 29(7):S166-S174

Ooms (2008) Digital holographic particle image velocimetry. PhD thesis, Delft University of Technology. Available at http://www. fm.3me.tudelft.nl/ thomas/Thesis/thesis.pdf

Ooms T, Koek W, Braat J, Westerweel J (2006) Optimizing Fourier filtering for digital holographic particle image velocimetry. Meas Sci Technol 17(2):304-312

Ooms T, Koek W, Westerweel J (2008) Digital holographic particle image velocimetry: eliminating a sign-ambiguity error and a bias error from the measured particle field displacement. Meas Sci Technol 19:074,003

Pereira F, Gharib M, Dabiri D, Modarress D (2000) Defocusing digital particle image velocimetry: a 3-component 3-dimensional DPIV measurement technique. Application to bubbly flows. Exp Fluids 29(7):78-84

Prasad A, Adrian R, Landreth C, Offutt P (1992) Effect of resolution on the speed and accuracy of particle image velocimetry interrogation. Exp Fluids 13(2):105-116

Satake S, Kunugi T, Sato K, Ito T, Taniguchi J (2005) Threedimensional flow tracking in a micro channel with high time resolution using micro digital-holographic particle tracking velocimetry. Opt Rev 121:442-444

Satake S, Kunugi T, Sato K, Ito T, Kanamori H, Taniguchi J (2006) Measurements of a $3 \mathrm{~d}$ flow in a micro-pipe via micro digital holographic particle tracking velocimetry. Meas Sci Technol 17:1647-1651

Sheng J, Malkiel E, Katz J (2006) Digital holographic microscope for measuring three-dimensional particle distributions and motions. Appl Optics 45:3893-3901

Sheng J, Malkiel E, Katz J (2008) Using digital holographic microscopy for simultaneous measurements of 3D near wall velocity and wall shear stress in a turbulent boundary layer. Exp Fluids

Stone H, Stroock A, Ajdari A (2004) Engineering flows in small devices. Annu Rev Fluid Mech 36(1):381-411

Westerweel J (2000) Theoretical analysis of the measurement precision in particle image velocimetry. Exp Fluids 29(7):3-12

Wong S, Ward M, Wharton C (2004) Micro T-mixer as a rapid mixing micromixer. Sensor Actuat B Chem 100(3):359-379

$\mathrm{Xu} \mathrm{L}$, Peng X, Miao J, Asundi A (2001) Studies of digital microscopic holography with applications to microstructure testing. Appl Optics 40:5046-5051

$\mathrm{Xu}$ W, Jericho M, Kreuzer H, Meinertzhagen I (2003) Tracking particles in four dimensions with in-line holographic microscopy. Opt Lett 28(3):164-166

Yamaguchi I, Zhang T (1997) Phase-shifting digital holography. Opt Lett 22(16): 1268-1270

Young I, Zagers R, Van Vliet L, Mullikin J, Boddeke F, Netten H (1993) Depth-of-focus in microscopy. Proc Scand Conf Image Anal 1:493-493 